\title{
UV Protection and Antibacterial Properties of Knitted Poly(Lactic Acid) Fabric Coated with Nano-ZnO
}

\author{
Jian-Yi Huang ${ }^{a}$, Kaiqiang Liu ${ }^{b}$, Jin-Ping Guan ${ }^{c}$ and Ren-Cheng Tang ${ }^{d}$ \\ National Engineering Laboratory for Modern Silk, College of Textile and Clothing Engineering, \\ Soochow University, 199 Renai Road, Suzhou 215123, China \\ a jianyi0808@163.com, b kliu@suda.edu.cn, \\ c guanjinping@suda.edu.cn, ${ }^{d}$ tangrencheng@suda.edu.cn
}

\begin{abstract}
Keywords: Poly(lactic acid); Fabric; Nano-ZnO; UV protection; Antibacterial activity.
Abstract. Nano-ZnO was successfully synthesized by a sol-gel method with an average size of 26.1 $\mathrm{nm}$. The nano-ZnO was applied to coat the knitted poly(lactic acid) (PLA) fabric with or without further coating using a polyacrylate binder, and the UV protection ability and antibacterial activity of the coated fabric were determined. The results showed that coating with nano- $\mathrm{ZnO}$ alone was not able to impart good UV protection ability and antibacterial activity to the fabric, indicating the poor affinity of nano-ZnO to PLA fiber. The nano- $\mathrm{ZnO}$ sol coating followed by binder coating was able to give very high UV protection factor (38.3) and antibacterial activity $(98.5 \%)$. The adhesive force of the binder contributed to the increased fixation of nano- $\mathrm{ZnO}$ on the fabric. In addition, the initial dosage of the binder had no significant impact on the UV protection ability of the fabric.
\end{abstract}

\section{Introduction}

In recent years, nano- $\mathrm{ZnO}$ has found its application in textile as UV blocker, antibacterial agent, fluorescent material, photo catalyst and so on [1]. The sol-gel method has been extensively used to synthesize nano-materials including nano- $\mathrm{ZnO}$ for its low cost, reliability, reproducibility, simplicity and relatively mild conditions of synthesis ("soft chemistry") [2]. Poly(lactic acid) (PLA) fiber has no aromatic ring [3], so it shows low absorbance and high transmittance to ultraviolet rays [4], which means good resistance to ultraviolet radiation. However, in the case of white or light color textiles, high transmittance of ultraviolet rays would do harm to human skin. This research aimed to improve the UV protection and antibacterial properties of PLA fabric with nano-ZnO. The nano-ZnO sol was first synthesized, and then used to coat PLA fabric by a pad-dry-cure process. Because nano-ZnO has no affinity to PLA fiber, a commercial polyacrylate binder was applied to improve the fixation of nano-ZnO on the fabric. In this work, the UV protection ability and antibacterial activity of the coated fabric were determined.

\section{Experimental}

Materials. The PLA fabric knitted using 9.74tex/2 yarn was kindly offered by Shandong Jide Ecological Technology Co. Ltd., China. Prior to use, the fabric was scoured in the solution containing $2 \mathrm{~g} / \mathrm{L}$ sodium bicarbonate and $0.5 \mathrm{~g} / \mathrm{L}$ nonionic surfactant to remove the spin oils. Zinc acetate dihydrate $\left(\mathrm{Zn}(\mathrm{Ac})_{2} \cdot 2 \mathrm{H}_{2} \mathrm{O}\right)$, ammonium citrate, anhydrous ethanol, and nitric acid $\left(\mathrm{HNO}_{3}\right)$ were analytical reagent grade chemicals. A commercially produced polyacrylate binder was used.

Preparation of Nano-ZnO Sol. The preparation of nano- $\mathrm{ZnO}$ sol was conducted according the previous report [5]. $15 \mathrm{ml}$ of aqueous $\mathrm{Zn}(\mathrm{Ac})_{2} \cdot 2 \mathrm{H}_{2} \mathrm{O}(0.01 \mathrm{~mol})$ solution was mixed at room temperature with $5 \mathrm{ml}$ of aqueous ammonium citrate $(0.03 \mathrm{~mol})$ solution followed by $20 \mathrm{ml}$ of ethanol which was added to form a white, cloudy suspension. Then the system was heated to $70{ }^{\circ} \mathrm{C}$. After that $\mathrm{HNO}_{3}$ was added dropwise until a transparent sol appeared. All the experiments were performanced under vigorous magnetic stirring. Afterwards the sol was aged in a hot-air oven at $70{ }^{\circ} \mathrm{C}$ to evaporate excess solvent and a concentrated sol was formed for later use. 
Fabric Coating. PLA fabric was immersed in the concentrated sol for $5 \mathrm{~min}$ and then padded in two dips and nips using a two-roll padder at a travel rate of $1 \mathrm{~m} / \mathrm{min}$ and a squeezing pressure of 2.4 $\mathrm{kg} / \mathrm{cm}$. The fabric was then dried at $80{ }^{\circ} \mathrm{C}$ for $10 \mathrm{~min}$ and cured at $130{ }^{\circ} \mathrm{C}$ for $3 \mathrm{~min}$. When further coated with 10-40 g/L binder, the fabric was treated with the same pad-dry-cure process again.

Measurements. The X-ray diffraction (XRD) analysis of nano-ZnO powder was conducted on the X'Pert-Pro MPD powder X-ray diffractometer. The particle size of the sol was measured by dynamic light scattering (DLS) using the Malvern Nano ZS 90 Zetasizer. The ultraviolet protection factor (UPF) and the ultraviolet (UV) transmittance of the fabric was determined in the Labsphere UV-1000F ultraviolet transmittance analyzer; each sample was tested four times at different positions, and the average value was recorded. The antibacterial property of the fabric was evaluated using the shake flask test according to GB/T 20944.3-2008 Textiles: Evaluation for antibacterial activity: Part 3 Shake flask method. The used bacterium was Escherichia coli. The reduction percentage of bacterium was calculated by Eq. (1):

$$
\mathrm{R}=100 \times(\mathrm{A}-\mathrm{B}) / \mathrm{A}
$$

where $\mathrm{A}$ is the number of bacteria colonies from the flask containing control cotton fabric after $18 \mathrm{~h}$ contact, and B is the number of bacteria colonies from the sample.

\section{Results and Discussion}

Characterization of Nano-ZnO Sol. The synthesized nano-ZnO sol had a homogenous distribution with an average particle size of $26.1 \mathrm{~nm}$ as shown in Fig. 1. After stored for several weeks, the nano-ZnO sol showed no precipitation.

The nano-ZnO sol was sufficiently dried at $150{ }^{\circ} \mathrm{C}$ in a hot-air oven. The residue was heated to 800 ${ }^{\circ} \mathrm{C}$ in $80 \mathrm{~min}$ and held $2 \mathrm{~h}$, and then cooled to ambient temperature. Finally, white nano-ZnO particles were obtained. The XRD pattern of the powdered sample is in Fig. 2. The nano-ZnO particles showed some reflections corresponding to (100), (002), (101), (102), (110), (103), and (112), which are matched to the standard diffraction pattern of wurtzite $\mathrm{ZnO}$ [6]. The particle sizes of $\mathrm{ZnO}$ calculated at the three main XRD peaks at $31.67^{\circ}(100), 34.31^{\circ}(002)$ and $36.15^{\circ}(101)$ using the Debye-Scherer formula (2) were 28,32 and $32 \mathrm{~nm}$, respectively.

$$
\mathrm{t}=0.89 \lambda /(\beta \cos \theta)
$$

where $\lambda$ is $X$-ray wavelength $(\lambda=0.15405 \mathrm{~nm}), \beta$ is peak width at half-maximum, $\theta$ is Brag diffraction angle.

The diameter of nano- $\mathrm{ZnO}$ particles calculated from the XRD is almost consistent with the particle sizes obtained from Fig. 2. Both of the DLS and XRD analyses indicate the successful preparation of the nano- $\mathrm{ZnO}$ sol.

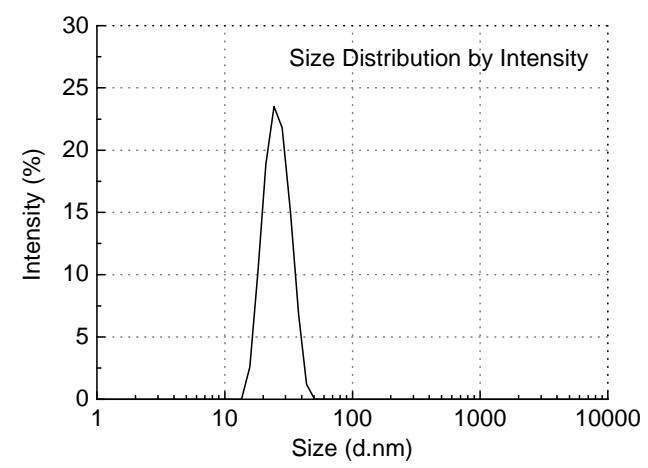

Fig. 1 DLS measurement of nano-ZnO sol

Coating with Nano-ZnO Sol. Taking the fact into consideration that nano-ZnO has no affinity to PLA fiber, a polyacrylate binder was used to combine these two materials. The sol is a stable 
electrical system and its stability would be broken by electrolyte. So the PLA fabric was treated with the concentrated sol and the binder separately instead of the mixture of them.

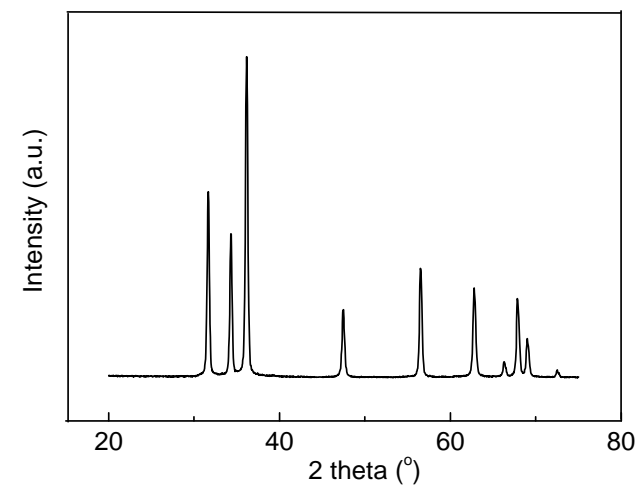

Fig. 2 XRD pattern of nano-ZnO powder

The fabrics were subjected to different treatments. The abbreviations of the related fabrics are listed in Table 1. Table 2 shows the UPF values and UV transmittance of the treated fabrics. The UPF value of the fabric increased from 7.04 to 38.30 after sol coating, binder coating and washing, while coating with sol only could give a slightly increased UPF. This implies the poor fixation of nano-ZnO on the fabric. Fig. 2 shows the UV transmission spectra of the fabrics. Compared with the untreated fabric, the fabric subjected to sol coating, binder coating and washing displayed significantly decreased UV transmittance. According to AS/NZS 4399-1996, the UPF values of less than 15, between 15 and 24, between 25 and 39, and above 40 are classified as bad, good, very good, and excellent protection against solar ultraviolet radiation, respectively [7]. The fabric subjected to sol coating, binder coating and washing exhibited very good UV protection ability.

Table 1. PLA samples prepared with various treatments

\begin{tabular}{llll}
\hline Sample & Sol & Binder & Washing \\
\hline Fab & No & No & No \\
Fab-SW & Yes & No & Yes \\
Fab-SBW & Yes & Yes & Yes \\
\hline
\end{tabular}

Table 2. UV transmittance and UPF of the coated fabrics

\begin{tabular}{llll}
\hline Sample & $T_{\text {UVA }}(\%)$ & $T_{\text {UVB }}(\%)$ & UPF \\
\hline Fab & 24.20 & 13.46 & 7.04 \\
Fab-SW & 18.50 & 8.05 & 10.84 \\
Fab-SBW & 8.96 & 1.98 & 38.30 \\
\hline
\end{tabular}

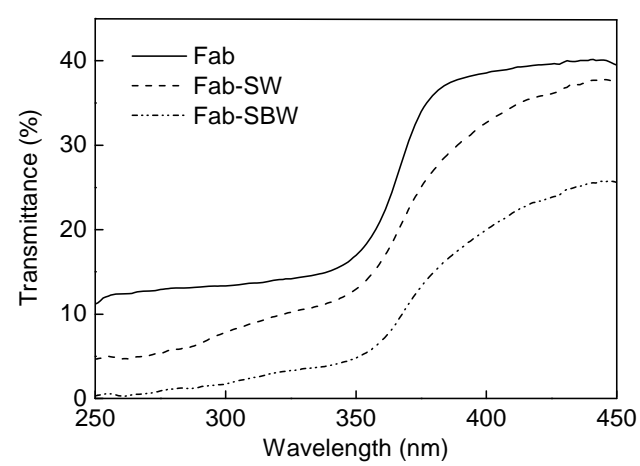

Fig. 3 UV transmittance spectra of the PLA fabrics subjected to various treatments

The antibacterial activity of the treated fabrics was evaluated by the reduction percentage of bacteria. After sol coating, binder coating and washing, the antibacterial activity of the fabric against 
E.coli increased from $0 \%$ to $98 \%$, while coating with sol only gave a very low antibacterial activity $(10.40 \%)$. This test also indicates that nano-ZnO has the poor affinity to PLA fiber, and binder coating can improve the extent of the fixation of nano- $\mathrm{ZnO}$ on the fabric by virtue of the binder's adhesive force. After sol and binder coating, the fabric exhibited very good antibacterial activity.

Effect of Binder Concentration on the UPF of the Fabric. The fabrics were subjected to sol coating, coating of binder at different dosages, and washing. The effect of binder concentration on the weight gain and UPF of the fabric was determined. Fig. 4 shows that the weight gain of the fabric linearly increased with increasing binder concentration while the UPF value increased to a limited extent. This suggests that there is no necessity to employ high binder concentration to get high functionality. In addition, high binder concentration can cause the hard handle of the fabric. Taking the functionality and handle of the treated fabric, the $20-30 \mathrm{~g} / \mathrm{L}$ dosage of nano-ZnO sol should be appropriate.

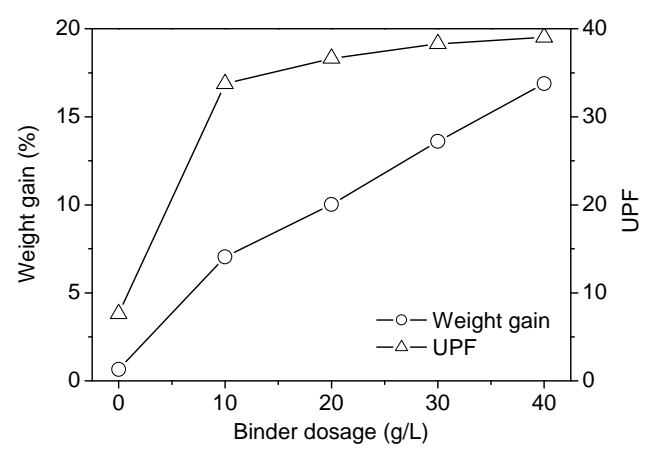

Fig. 4 Weight gain and UPF of the fabrics coated by nano-ZnO sol followed by binder coating

\section{Conclusions}

The nano-ZnO sol was successfully synthesized with a homogenous distribution and an average size of $26.1 \mathrm{~nm}$. The obtained sol was applied to treat the knitted PLA fabric by a pad-dry-cure process. The UV protection capability and antibacterial activity of the fabric were slightly improved by coating only with sol, and significantly increased by sol coating followed by binder coating. Binder coating was able to increase the fixation of nano- $\mathrm{ZnO}$ on the fabric, and remarkably improve the functional properties of the treated fabric.

\section{Acknowledgements}

This work was financially supported by Jiangsu Provincial Natural Science Foundation of China (BK2012197), Joint Innovation Fund of Jiangsu Province of China (BY2014059-04), and Suzhou Science and Technology Support Program (SS201422).

\section{References}

[1] B.A. Çakir, L. Budama, Ö. Topel and N. Hoda: Colloid. Surface. A, Vol. 414 (2012), p. 132

[2] J. Lee, A. J. Easteal, U. Pal and D. Bhattacharyya: Curr. Appl. Phys., Vol. 9 (2009), p. 792

[3] J. Lunt and A.L. Shafer: J. Ind. Text., Vol. 29 (2000), p. 191

[4] K. Mogi, H. Kubokawa, H. Komatsu and T. Nakamura: Sen'i Gakkaishi, Vol. 59 (2003), p. 198

[5] Y. Cong, G.L. Ning, X. Huang and B.L. Lv: Chin. J. Sci. Instrum., Vol. 16 (1995), p. 309

[6] M. Sato, A. Kawata, S. Morito, Y. Sato and I. Yamaguchi: Eur. Polym. J., 44 (2008), p. 3430

[7] S.S. Sun and R.C. Tang: Ind. Eng. Chem. Res., 50 (2011), p. 4222 